$$
\begin{gathered}
\text { COAF }-9009508 \cdots-4 \\
\text { UCRL-JC- }-105534
\end{gathered}
$$

DE 91005430

UCRL- JC 105534

PREPRINT

THE ORIGIN AND DEVELOPMENT OF INSTABILITIES DEC 871990
IN RADIATIVELY-DRIVEN STELLAR WINDS

JOHN I. CASTOR

THIS PAPER WAS PREPARED TO BE PRESENTED TO

THE NATO ADVANCED STUDY WORKSHOP "STELLAR

ATMOSPHERES: BEYOND CLASSICAL MODELS"

TRIESTE, ITALY

SEPTEMBER $3-7,1990$

NOVEMBER $20,1.990$

This is a preprint of a paper intended for publication in a journal or proceedings. Since changes may be made before publication, this preprint is made available with the understanding that it will not be cited or reproduced without the permission of the author.

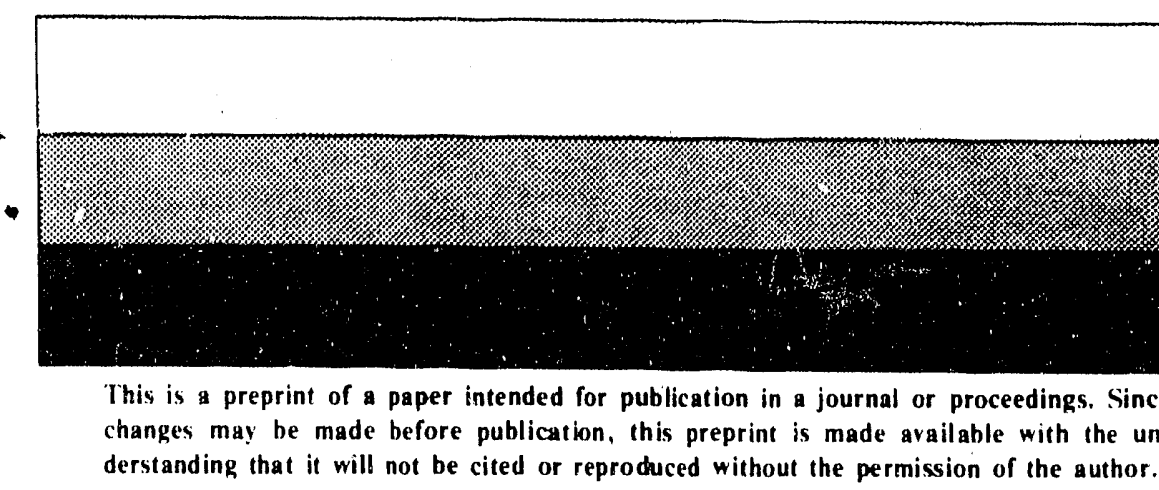

OASTRIBUTION OF THIS DOCUMENT \& IJHLIMITEO 


\section{DISCLAIMIER}

This document was prepared as an account of work sponsored by an agency of the I'nited States Government. Neither the linited States Government nor the liniversity of Califorsia nor any of their employees, makes any warranty. express or implied, or assumes any legal liability or responsibility for the accuracy, completeness, or usefulness of any information. apparatus, product, or process disclosed, or represents that its use nould not infringe privately owned rights. Reference herein to any specific commercial products, process, or service by trade name. "ademark. manufacturer. or utherwise, does not necessarily constitute or imply its endorsement, recommendation. or favoring by the I'nited States Government or the l'niversity of California. The viess and opinions of authors expressed herein do not necessarily state or reflect those of the Lnited States Government or the University of California, and shell noi be used for advertining or product endorsement purposis. 


\title{
THE ORIGIN AND DEVELOPMENT OF INSTABILITIES IN RADIATIVELY-DRIVEN STELLAR WINDS*
}

\author{
JOHN I. CASTOR \\ Laurence Livermore National Laboratory \\ Livermore, CA 94550 \\ U. S. A.
}

\begin{abstract}
The numerous observational indicators of instability in the radiatively-driven winds of hot stars are reviewed briefly, followed by a summary of the present theoretical understanding of the linear instability of such winds. This provides the motivation sor the hydrodynamic simulations, the major thrust of the paper. A serious approximation that must be made in order to reduce the cost of the simulations to a reasonable level-the absorption approximation for the radiation force-is discussed in some detail. The hydrodynamic methods are described briefly, and then the computational results for winds models computed in the absorption approximation are discussed. The most notable results pertain to the critical nature of the ratio $v_{\text {th }} / a$ of the intrinsic line width to the sound speed. When this ratio is large, only a negligible wind results; when the ratio is small, the wind executes permanent self-excited oscillations; in an intermediate range the wind is globally stable, but acts as a powerful wave amplifier. The morphology of the oscillations-strong rarefactions and reverse shocks-is described and related to Abbott's linear theory, and the possible connection to observations is mentioned.
\end{abstract}

\section{Introduction}

The theory of the intense winds emanciing from the luminous $\mathrm{O}$ and $\mathrm{B}$ stars has made a great deal of progress since the winds were first discovered in the UV spectra of the Orion Belt stars (Morton 1967). The theory of a steady, radiatively-driven wind (Lucy and Solomon 1970; Castor, Abbott and Klein 1975 [referred to below as CAK]; Friend and Abbott 1986; Pauldrach, Puls and Kudritzki 1986) has been quite successful and is now able to explain many features of the winds, quantitatively and without adjustable parameters. The steady models fail at explaining certain other features-the fluctuating absoption in some of the UV lines, the presence in the spectrum of lines of nigh-ionization species like O VI, the $x$-ray emission, the blackness of the UV absorption troughs. These features point to the presence of a chaotic velocity field. The steady radiatively-driven wind is now known to be very unstable (Lucy and Solomon 1970; MacGregor, Hartmann and Raymond 1979; Owocki and Rybicki 1984, 1985), and most people believe that this is the origin of the chaotic velocities. The high-ionization species may well owe their presence to the $x$-rays (Cassinelli and Olson 1979), and the x-rays may well be produced in shock fronts within

* This work was performed in part under the auspices of the U. S. Department of Energy by the Lawrence Livermore National Laboratory under Contract No. W-7405-ENG-48. 
the wind that are inevitable given the chaotic velocities (Lucy and White 1980; Lucy 1982). However, observations have not so far provided a clear kinematic picture of this chaos, and the rather simple pictures provided by the theory to date have not fared well in comparisons with the data ( $c f$, Cassinelli and Swank 1983).

The present work describes a project to illuminate the morphology of the chaotic wind by means of direct hydrodynamic simulations. This is perhaps a foolhardy venture, since the winds are no doubt more complicated than we can even imagine, let alone calculate; yet there seems to be no other way to proceed. An observational approach, in the manner of solar physics, is severely limited by the lack of spatially-resolved data. The numerical simulations are certain to be inaccurate at best, and occasionally will contain major qualitative errors. Their value lies in their use as a catalog of possible morphologies of the wind; they can serve as guides to the interpretation of the observational data. As the models are elaborated and refined, it may be hoped that they will eventually become realistic pictures of the true state of the wind.

This paper will provide a little background on the radiation-driven wind instability, explain the reasons for making a certain severe approximation to the radiation force, provide some description of the numerical hydrodynamics methodology, and finish with a discussion of the first set of computational results. This material has been published by Owocki, Castor and Rybicki (1988) and Poe, Owocki and Castor (1990). The more recent simulations that have relaxed, to some degree, the severe approximation just mentioned, are discussed by Owocki in the following paper in this Workshop.

\section{Why Radiatively-driven Stellar Winds are Unstable}

The mechanism of the instability was first suggested by Milne (1926), mentioned also in the seminal paper by Lucy and Solomon (1970), and confirmed in the later work. The idea is simple: The force that drives the wind is due to spectral line absorption, primarily in the strong resonance lines. The absorbing atoms are self-shielding-enough fluid is moving at any given velocity that the spectrum of the intensity absorbed by a typical atom has a strong absorption feature due to the other atoms that lie in the path of the radiation. Because of this absorption, the total force on the fluid is a small fraction of the value that would result if the absorbing atoms were exposed to a flat continuous spectrum. If now a small parcel of fluid is perturbed in velocity, the force on the parcel changes dramatically. If the perturbation is positive, the frequency at which the atoms in the parcel absorb is shifted toward the clear part of the spectrum, and the force rises; a negative perturbation shifts the absorption frequency into a region where the attenuation of the continuum is even larger, and the force drops.

The mathematical development of the instability proceeds as follows. The Euler equation is modified by the addition of the radiation force $\rho \mathrm{g}_{\mathrm{rad}}$ and gravity:

$$
\rho \frac{\partial \mathbf{v}}{\partial t}+\rho \mathbf{v} \cdot \nabla \mathbf{v}=-\nabla \mathbf{p}+\rho \mathbf{g}_{\mathrm{rad}}-\rho \mathbf{g}
$$

The radiative acceleration $\mathrm{g}_{\mathrm{rad}}$ is a functional of the velocity, and the instability of the wind is governed by its variational derivative with respect to the velocity, the tensor operator $\delta \mathbf{g}_{\text {rad }} / \delta \mathbf{v}$. As the physical argument in the preceding paragraph suggests, this operator may have large positive eigenvalues, $\gamma$, which lead to growth of the perturbation as $\exp (\gamma t)$. It 
will be seen shortly that the largest $\gamma$ is $O\left(g_{\text {,ad }} / v_{\text {th }}\right)$, which is also $O\left(\left(v / v_{\text {th }}\right) d v / d r\right)$. The number of linear e-folds that a perturbation could receive during a wind flow time is $\int \gamma d r / v$, which is therefore $O\left(v_{\max } / v_{\text {th }}\right) \approx 300$ !

The radiative acceleration is the sum of parts due to continuous absorption and scattering and a part due to lines. The continuum absorption part is usuilly negligible, while the continuum scattering part is dominated by electron scattering, so its contribution to $\mathrm{g}_{\mathrm{rad}}$ is

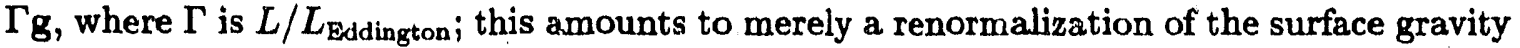
by a factor $1-\Gamma$. The line part of $\mathrm{g}_{\mathrm{rad}}$ is

$$
\mathrm{g}_{\text {line }}=\sum_{\ell} \mathrm{g}_{\ell}=\sum_{\ell} \frac{\kappa_{\ell} \Delta \nu_{\mathrm{D}}}{c} \iint \Omega d \Omega \phi(x) d x I(\Omega, \nu) .
$$

The variable $x$ is the frequency offset from the local fluid-frame line center, in units of the Doppler width $\Delta \nu_{D}=\nu_{0} v_{\text {th }} / c_{\text {n }}$ The observer's-frame frequency $\nu$ in the integral is to be expressed in terms of $x$ and the direction vector $\Omega$ by $\nu=\nu_{0}+\Delta \nu_{D}\left(v \cdot \Omega / v_{\text {th }}+x\right)$. Evidently the velocity sensitivity of $\mathrm{g}_{\mathrm{rad}}$ is due to the spectral shape of $I$.

In the wind, where the velocity is hypersonic, the Sobolev length $\mathcal{L} \equiv v_{\text {th }} /|\Omega \cdot \nabla v \cdot \Omega|$ is small compared with the scale lengths of density and velocity. Points much farther apart than $\mathcal{L}$ can not communicate through spectral line transfer, so when $\mathcal{L}$ is small a local approximation, the Sobolev approximation, becomes accurate. The optical depth can then be calculated knowing only the local opacity and the Sobolev length. The transfer equation yields the intensity in terms of the local source function $S$ and the photospheric continuum intensity $I_{c}$ :

$$
I=I_{c} \exp \left(-\tau_{S} \Phi(x)\right)+S\left(1-\exp \left(-\tau_{S} \Phi(x)\right)\right),
$$

where the Sobolev optical depth $\tau_{S}$ is $\chi_{\ell} \mathcal{L}$ and $\Phi(x)$ is the indefinite integral of the profile function, $\Phi(x)=(1 / 2) \operatorname{erfc}(x)$. The $I_{c}$ term in $I$ is omitted unless the direction in question is outward-directed and its backward extension intersects the photosphere. The Sobolev optical depth is the same for oppositely-directed rays, so in the Sobolev approximation the force derives entirely from the direct part, i.e., the $I_{c}$ term. If the Sobolev approximation (3) for $I$ is combined with equation (2), it is seen that the Sobolev force is a function of the local strain tensor $\left(\nabla \mathbf{v}+(\nabla \mathbf{v})^{\mathrm{T}}\right) / 2$.

The linear stability analysis of equation (1) with the Sobolev approximation, made by Abbott (1980), leads to waves with speeds quite modified from ordinary sound waves, but not to a strong instability. The apparent contradiction between this result and that of MacGregor, et al. is resolved by noting the different assumptions implicitly made about the ordering of the scale length of the velocity perturbation and $\mathcal{L}$. For Sobolev to be valid $\mathcal{L} \ll$ scale length. The MacGregor, et al. picture assumes that the intensity is unperturbed in the rest frame of the unperturbed fluid, which requires that $\mathcal{L} \gg$ scale length. These two analyses were unified by Owocki and Rybicki $(1984,1985)$. With the replacement of the sum over lines by a statistical ensemble, $d N(\chi) \propto \chi^{\alpha-2} d \chi$, as in Castor, et al. (1975), Owocki and Rybicki found that a sinusoidal velocity perturbation $\sim \exp (i k r)$ gives a force perturbation

$$
\frac{\delta \mathbf{g}_{\mathrm{rad}}}{\delta \mathbf{v}} \approx \frac{\mathbf{g}_{\text {line }}}{v_{\text {th }}} \frac{i k \mathcal{L}}{1+i k \mathcal{L}}
$$

which they called the "bridging law." The large- $k$ limit of the bridging law recovers the MacGregor, et al. result of strong instability, and the small- $k$ limit reproduces Abbott's 
wave mocies with no instability. If the small- $k$ (or small $\mathcal{L}$ ) expansion of equation (4) is carried to the $(k \mathcal{L})^{2}$ term, however, it is seen that a viscous-like force term comes into the Euler equation, except that the equivalent kinematic viscosity is the negative quantity $\nu \approx-\mathrm{g}_{\text {line }} \mathcal{L}^{2} / v_{\text {th }}$. Thus it should be no surprise that the flow is unstable even in the long-wavelength régime.

\section{The Absorption Approximation}

It was noted in the previous section that the $S$ term in the intensity, the diffuse term, makes no contribution to the force in the Sobolev approximation. What will be called the absorption approximation in the remainder of this paper, and in Owocki's paper to follow, is to continue to neglect the diffuse force, but to calculate accurately the force due to the $I_{c}$ term, without making the Sobolev approximation. Owocki and Rybicki (1984) obtained the bridging law (4) from the direct force. This is the approximation used for the numerical simulations to be described shortly, but some consideration of the consequences of the absorption approximation is appropriate now.

The diffuse intensity is responsible for an important counter term in the perturbed force for the short-wavelength limit. The force perturbation in that limit is easily recovered from equation (2) by perturbing the argument $x$ of $\phi(x)$ by $\Omega \cdot \delta \mathrm{v} / v_{\mathrm{th}}$, while continuing to use equation (3) for the unperturbed intensity. Because the frequency profile of the diffuse intensity is just inverted from that of the direct intensity, its force perturbation is opposite. With the usual definition $\mu=\Omega_{r}$, it is apparent that the diffuse force perturbation is even in $\mu$ over the range $-1 \leq \mu \leq 1$, while the direct force perturbation arises only from $\mu \geq \mu_{*}=\left(1-\left(R_{*} / r\right)^{2}\right)^{1 / 2}$. The proportion of diffuse force perturbation to direct force perturbation is therefore roughly as $S: W I_{c}$, where $W$ is the dilution factor $\left(1-\mu_{*}\right) / 2$. Since for a conservative-scattering line the source function is approximately $W I_{c}$, the force perturbation nearly cancels out. This is essentially the argument presented by Lucy (1984), who refers to the diffuse force perturbation as the "drag effect." The more accurate evaluation of the diffuse force perturbation by Owocki and Rybicki (1985) showed that the force perturbation remains positive, though diminished by a large factor for $r \rightarrow R_{*}$.

The same kind of simple argument that can be used to derive the bridging law (4) for the direct force perturbation leads to

$$
\frac{\delta \mathbf{g}_{\text {diff }}}{\delta \mathbf{v}} \propto-\frac{(k \mathcal{L})^{2}}{1+(k \mathcal{L})^{2}}
$$

which demonstrates the stabilizing effect of the diffuse force in the short-wavelength limit, and also indicates that the diffuse force introduces positive viscosity in the long-wavelength limit; both effects offset aspects of the direct force.

Since some important consequences of the diffuse force have just been brought forward, why have the calculations been made in the absorption approximation? The answer is that the absorption approximation is a well-defined approximation that is relatively cheap to calculate. "Relatively cheap" means that a single model can be evolved long enough to determine its behavior in from one to several hours of Cray CPU time. The extra cost for replacing the absorption approximation with the calculation of a few representative scattering lines in detail is estimated to be at least $50 \times$. (A factor 5 for the number of lines to consider, and a factor of more than 10 for the number of $\mathrm{AOI}$ iterations to treat 
the scattering and for the extra algebra of a full formal solution.) This cost has so far been considered to be prohibitive.

For the numerical evaluation of $g_{\text {ine }}$ equation (2) is used, with $I$ evaluated from the absorption approximation, $I_{\nu}(\mu)=I_{c}(\mu) \exp (-\kappa \eta(r, \mu, x))$, and with the summation over lines replaced by an integration over the power-law ensemble mentioned above. (The symbol $\mu$ is used for the angle variable, but in reality a ray with a certain impact parameter $p$ is intended, where, at a given $r, \mu$ is related to $p$ by $\mu=\sqrt{1-p^{2} / r^{2}}$; the notation $\mu$ will be retained for simplicity.) Here $\eta(r, \mu, x)$ is the profile-weighted mass column density between $R$. and $r$ :

$$
\eta(r, \mu, x)=\int_{R_{.}}^{r} d z\left(r^{\prime}\right) \rho\left(r^{\prime}\right) \phi\left(x+\mu \frac{v(r)}{v_{\text {th }}}-\mu^{\prime} \frac{v\left(r^{\prime}\right)}{v_{\text {th }}}\right)+m_{0} \phi\left(x+\mu \frac{v(r)}{v_{\text {th }}}\right) .
$$

The term in $m_{0}$ accounts for an absorption profile assumed to be produced in the photospheric spectrum by the line in question, approximated by a Schuster-Schwarzschild model for which $m_{0}$ is the reversing-layer thickness. If $m_{0}$ were omitted the force would be unreasonably large for $r \rightarrow R_{*}$. The line force then becomes

$$
\begin{aligned}
g_{\text {line }} & =\int_{0}^{\infty} d \kappa N_{0} \kappa^{\alpha-2} \exp \left(-\frac{\kappa}{\kappa_{\text {cut }}}\right) \frac{2 \pi \kappa v_{\text {th }}}{c^{2}}\left\langle\frac{\nu F_{\nu}}{F}\right\rangle \int_{\mu \cdot}^{1} \mu d \mu \int_{-\infty}^{\infty} d x \phi(x) I_{c} \exp (-\kappa \eta(r, \mu, x)) \\
& =2 \pi N_{0} \frac{v_{\text {th }}}{c^{2}}\left\langle\frac{\nu F_{\nu}}{F}\right\rangle \Gamma(\alpha) I_{c} \int_{\mu .}^{1} \mu d \mu \int_{-\infty}^{\infty} d x \phi(x)\left[\eta(r, \mu, x)+\kappa_{\text {cut }}^{-1}\right]^{-\alpha} \\
& \approx N_{0} \frac{v_{\text {th }}}{c^{2}}\left\langle\frac{\nu F_{\nu}}{F}\right\rangle \Gamma(\alpha) \frac{L_{*}}{4 \pi r^{2}} \int_{-\infty}^{\infty} d x \phi(x)\left[\eta(r, \mu, x)+\kappa_{\text {cut }}^{-1}\right]^{-\alpha}
\end{aligned}
$$

The exponential factor involving $\kappa / \kappa_{\text {cut }}$ is essential, since otherwise when the velocity gradient becomes steeper and steeper, the force would increase without limit. The cutoff accounts for the reality that the total oscillator strength of the line ensemble has a finite value. The last approximate equality in (7) comes from the choice of a single average ray (designated by $\mu$ ) to represent the angle integration. (The ray with $p=R_{*} / \sqrt{2}$ turns out to give an excellent approximation.) Almost all the CPU time for the calculation is spent on the integration in equation (6); for this reason the integration is highly optimized and makes use of a fast, modest-accuracy routine for the complementary error function, $2 \Phi(x)$.

\section{Numerical Hydrodynamics Techniques}

The goal of the hydrodynamic simulations is to study the instability of the radiativelydriven wind by direct time integration of the fluid equations. Numerous simplifications and approximations are made, including:

1. spherical symmetry

2. absorption approximation

3. isothermal

4. $\alpha$-power line ensemble; individual $\kappa_{\ell} s$ constant; no ionization effects

5. modified point-source model

6. magnified $a$ and $v_{\text {th }}$ (by $\sqrt{10}$ here) to ameliorate multi-scale problem

Some of these are reasonable approximations; others, like the absorption approximation, are needed to make an impossible problem feasible. 
The isothermal approximation warrants some additional comment. To compute the hydrodynamics isothermally does not mean that there can not be any hot material in the wind; this would rule out the likeliest mechanism for producing $x$-rays. It means that such hot material as there may be should be concentrated in thin regions behind shock fronts. The numerical calculation "skips over" such layers, but, since the Rankine-Hugoniot shock jump conditions are obeyed both in nature and in the code, the large scale hydrodynamics of the wind is computed correctly. There is a condition that must be met for the hot layer to be thin ( $c f$. , Castor 1987), which is that the post-shock cooling length should be smaller than the distance between shocks. When expressed as a column density, the cooling length is about $9 \times 10^{20} \mathrm{~cm}^{-2}$ for a $600 \mathrm{~km} \mathrm{~s}^{-1}$ shock velocity, while the column density between shocks is roughly $10^{22} \dot{\mathcal{M}}_{6} \mathrm{~cm}^{-2}$, where $\dot{\mathcal{M}}_{6}$ is the mass loss rate in the wind in units of $10^{-6} \mathcal{M}_{\odot} \mathrm{y}^{-1}$. Thus isothermality should be an adequate approximation for $\dot{\mathcal{M}}>10^{-7} \mathcal{M}_{\odot} \mathrm{y}^{-1}$, which is satisfied for the more luminous $\mathrm{O}$ stars and $\mathrm{B}$ supergiants.

The salient features of the hydrodynamic method are these: explicit time-stepping; a fixed Eulerian spatial mesh; von Neumann and Richtmyer staggering in space and time; operator splitting-time advancement is done separately for the application of forces, energy balance, mass and energy advection, and momentum advection; van Leer monotonic piecewise-linear interpolation (van Leer 1977) is used to calculate advection; von NeumannRichtmyer quadratic pseudoviscosity; time step control based on Courant-Friedrichs-Lewy condition combined with constraints on density change and instability growth.

The spatial zoning is set up with a zone size that provides a few zones per Sobolev length, if the run of velocity is relatively smooth; in those cases in which sharp gradients appear (other than shocks) there may then be only one zone per Sobolev length in the gradient region. Because of the large value of $v_{\max } / v_{\text {th }}$, several hundred spatial zones must be used. With the customary time-step constraints it may require several thousand time-steps to advance by one flow time for the outer part of the wind, and several tens of thousands of time-steps for the flow to relax to a steady state, or to a quasi-periodic oscillation. The temptation to coarsen the spatial mesh, or to use an implicit time-stepping method with large steps, is great, but to do so would do great violence to the description of an instability that is strongest for these very small spatial and temporal scales.

The hydrodynamic boundary conditions for the wind calculation are these: At the outer boundary, which may, by necessity, not be as remote as desired, the boundary condition should allow waves to pass freely outward without reflection. The boundary should be in a region where the outward flow velocity is always supersonic, and therefore no characteristics ( $c f$. , Courant and Friedrichs 1948) enter the solution domain from outside. Computationally, the needed data in the phantom zone beyond the boundary are obtained by extrapolation from within. At the inner boundary, where the flow is subsonic, the condition is that, again, waves should pass freely out of the solution domain without reflection. In this case the $C_{+}$characteristic enters the domain at the boundary, and the corresponding Riemann invariant should be specified (Hedstrom 1979). This boundary condition will be applied at the radius of the mean photosphere, and therefore should also serve to fix the density at the photospheric value in the absence of waves. These objectives are achieved by the relation $v+a \ln \left(\rho / \rho_{0}\right)=0$, in which $a$ is the speed of sound and $\rho_{0}$ is the nominal photospheric density. The case that a flux of sound waves is incident on the wind from below the photosphere is treated by replacing 0 on the right side of this condition with the driving function $2 A \cos (\omega t)$.

The radiatively-driven stellar wind has, as discussed by Owocki and Rybicki (1986), a very powerful advective instability in the sense of Bers (1983, who refers to this as convective 
instability); that is, it amplifies a perturbation by a large factor as the perturbation is dragged away from the star by the flow. The wind may or may not have an absolute instability, in which the residual disturbance near the location of the perturbation is amplified with time. In the former case the wind is an amplifier of perturbations; in the latter case it becomes a self-excited oscillator, and the steady flow is inevitably disrupted. Owocki and Rybicki (1986) showed that the supersonic region is stable in the absolute sense, but the question remains open for the subsonic region. One objective of the numerical work is to determine whethes there is an absolute (or global) instability. Just as even poor mirrors can transform a laser amplifier into a laser osciliator, any wave reflection at the boundaries due to numerical errors can lead to a spurious inference of global instability; the same can be said of improper spatial centering of the radiation force and even the action-at-a-distance produced by modulation of the adaptive time step! All these factors must be carefully controlled to avoid false instabilities.

A fundamental problem of calculations such as these is that it is impossible, in principle, to obtain an accurate answer. The existence of unresolved spatial scales with their very large instability growth rates makes the initial-value problem decidedly ill-posed. This difficulty is shared with simulations of turbulent flow, thermal convection, the earth's weather, etc., although the growth rate of the instability is much greater in the present case. The only valid results that can be obtained are statistical, and these may be sensitive to the fineness of the mesh.

\section{Results of Hydrodynamic Calculations}

The most notable result of the hydrodynamic wind simulations in the absorption approximation is that both global stability and instability are observed, with the critical parameter being the ratio of the thermal line width to the sound speed, $v_{\mathrm{th}} / a$. Specifically, the following behavior is found:

$$
\begin{array}{ll}
v_{\text {th }} / a \geq 0.5 & \rightarrow \text { negligible wind } \\
0.35 \leq v_{\text {th }} / a \leq 0.5 & \rightarrow \text { steady wind } \\
v_{\text {th }} / a<0.35 & \rightarrow \text { strong waves permanently present }
\end{array}
$$

The reason for this strong sensitivity to the value of $v_{\text {th }}$ is the absorption profile in the photospheric spectrum, viz., the $m_{0}$ term in equation (6). With the value of $m_{0}$ used for the simulations, and for a given mase flux, the absorption force is very small until $v \gtrsim 2 v_{\text {th }}$. Thus in the first case above, $v_{\text {th }} / a \geq 0.5$, the radiation force is too small at the sonic point to permit a smooth transonic fiow unless the mass flux is very low. In faci, this is another unfortunate consequence of the absorption approximation. In the intermediate range of $v_{\text {th }} / a$ the value of $v / v_{\text {th }}$ near the sonic point is large enough for the force to be determined by the relatively unblackened part of the photospheric spectrum, and a sizeable (i.e., comparable to CAK) mass flux results. As the thermal velocity is further reduced, the gross amount of amplification of the advective instability up to the sonic point, which scales as $a / v_{\text {th }}$, becomes large enough that an absolute instability is apparently produced in the subsonic region.

The physical effect of $v_{\text {th }} / a$ just discussed is also responsible for changes in the topology of the sonic point in the steady flow models. As discussed by Poe, Owocki and Rybicki (1990), the sonic point is a node for wind models with the absorption force, not the familiar 


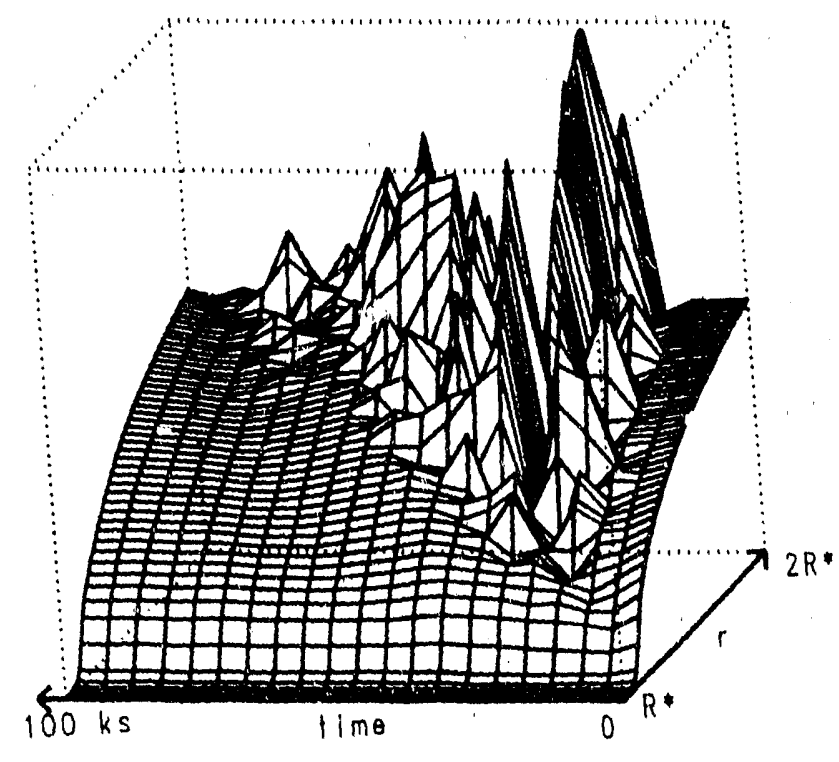

Figure 1: Perspective plot of flow velocity vs. radius and time for a wind with $v_{\text {th }} / a=3 / 8$. The ther data for the model follow Owocki, Castor and Rybicki (1988).

Parker-type saddle point. The node has two positive critical values of $d v / d r$, and there is Pn infinite family of solutions through the sonic point with the smaller critical slope. The an infition be nodal topology and the stability behavior of the wind is discussed by Owocki in the next paper of this Workshop. Both the very low force for $v \lesssim 2 v_{\text {th }}$ and the large approximation. Owocki, in the next subsonic region are quite sensitive to the absorption an estimate of the diffuse, scattering paper, will show how these results are che $v_{\text {th }} / a=3 / 8$. The
force is included.
Figure 1 shows the time evolution of the run of velocity for a wind with

Figure 1 shows the time evolutimilar to, but not identical with, the steady flow. As seen in initial state was chosen i.e., no oscillation was applied at the inside boundary. As seen waves the model was undriven, of the initial state from the true steady solution ploduct times the the figure, the departure of the they proceed outward. But after about two flow final state which are strongly ample and any further calculation leads to no change; the focted a wind becomes quiescent, and to the steady one. This is just the behavior The that predicted by the The steady structure found in this calculation is qualitatively while the velocity is within CAK theory. The mass loss rate is one third less the tocation predicted by the CAK about $10 \%$ at $r=2 R$. The sonic point is very close to velocity gradient at the sonic point theory (consistent with the agreement of $\mathcal{M}$ ), but this is the diminution of the force by the is a few times larger than CAK. The reason for this is the diming a steeper gradient.

photospheric absorption profile, which is compentedendent behavior is quite different, When $v_{\text {th }} / a$, has the smaller value 0.255 , the then run, of order $3 \times 10^{5} \mathrm{~s} \approx \mathrm{six}$ as shown in Figure 2. For as long as this calculation has been rin, 


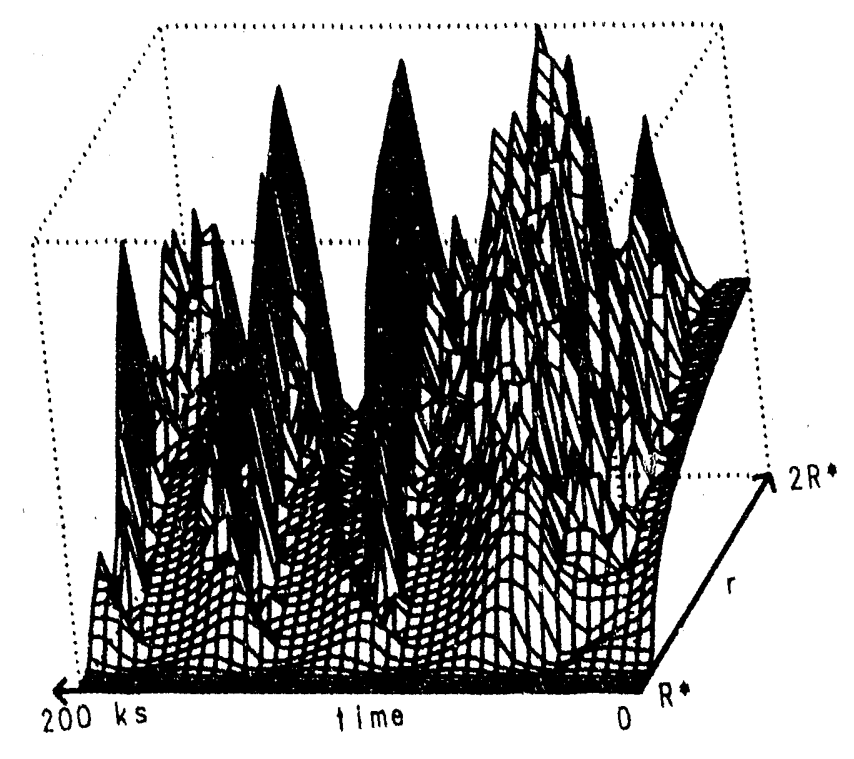

Figure 2: Like Fig. 1, but with $v_{t b} / a=0.255$. waves. The oscillations are quasiperiodic, in that much of the power in the temporal pow the spectrum is concentrated around a period of $4.5 \times 10^{4} \mathrm{~s}$. This ponic point. Since this is the acoustic cut-off period of the atmosphere, $4 \pi a / 9$, naps not surprising that it dominates the natural ringing period of the atmospher self-excited wave spectrum.

The waves that one sees prome in the $v_{\text {th }} / a=0.255$ model, are lasing, since the growth and which are permanently prefactions. The long wavelength is surp ate only for wavelengths relatively long-wavelength raches half of the maximum grew to 20 percent of $R$. Thus the rate of the linear instabiling on the radius, this is a few percent the upper limit of the range for wavelengths that dominate the simulation are at or best. which the linear instability is strongest. The rarsfaction nature of the waves is dh rise in velocity with in by a smooth decrease in has a sawtooth-like part, with a sharp drop. This is accompanied thucture is inward-facing, on the large-radius side by a rise where the velocity drops. This stiarp drop on the cuter density followed by a sharp rise wh the inner side and exits at the material, it always travels i.e., matter flows into the wave at thels inward relative to the mass in velocity and density side. Although the wave thume of the star. The sharp jumps in veck. In a certain range outward relative to the fixed fre it faces inward, is called a reverse shous, by a lorward shock. mark a shock front, which, since it fampanied, just outside it in side. Therefore the region of radius the reverse shock is ack wave from the larger radius the reverse sheck as well as The material flows into this shock wave matter compressed by the reverse sheck as well as between the two shocks accumulates the matter 
the matter compressed by the forward shock. This dense region can become rather thin, and yet contain a large part of the total mass column density of the wave structure.

It is tempting to identify the dense shells associated with the reverse-forward shock pairs as the origin of the narrow absorption components observed in the P Cygni profiles of UV resonance lines in the spectra of many OB stars (Lamers, Gathier and Snow 1982; Henrichs 1984; Prinja and Howarth 1986, 1988). In this picture the typical velocity of the inter-shock gas is identified with the velocity of the narrow components, while the highest velocity of the gas in the rarefaction is identified with the blue-edge velocity of the $\mathrm{P}$ Cygni profile. The low density of the highest velocity gas provides an explanation for the often-observed softness and variability of the blue edge. The velocity jumps across the shocks are a few hundred $\mathrm{km} \mathrm{s}^{-1}$, of just the right order to account for the $\mathrm{x}$-ray emission (Cassinelli and Swank 1983).

The major conflict between this picture and observations concerns the time scale for acceleration and change of the absorption components. As found by Lamers et al. (1988), Kaper et al. (1990) and Prinja (1990), the time scale for a narrow component to progress through its range of velocity ranges from half a day to several days, significantly longer than the flow time, and furthermore that time and the recurrence time of the components evidently scale with the rotation period of the star. It is highly likely that the spherically symmetric model of the instability omits important 3-D effects, and that surface inhomogeneities of the star play an important role in exciting and phase-locking the waves.

Because the wavelengths of the prominent waves in the simulations are rather larger than $2 \pi \mathcal{L}$, it is useful to describe them in terms of the wave modes discussed by Abbott (1980). Abbott took the Sobolev approximation, for which, as already noted, the radiation force is a function of the local strain tensor. In the simple case of radial flow and a point source of radiation, ghine depends on $d v / d r$; its derivative defines a characteristic. speed, $U=\partial g_{\text {ine }} / \partial(d v / d r)$. The speed $U$ is related to the Sobolev length $\mathcal{L}$ and the optically-thin growth rate $g_{\text {line }} / v_{\text {th }}$ by $U \approx g_{\text {line }} \mathcal{L} / v_{\text {th }}$. The dispersion relation obtained from the Euler equations including $g_{1 \text { line }}$ leads to two wave modes, one with phase velocity $v_{p}=v-\left(U+\sqrt{U^{2}+4 a^{2}}\right) / 2$ which will be called the inward mode, and one with $v_{p}=v+\left(-U+\sqrt{U^{2}+4 a^{2}}\right) / 2$, the outward mode. The designation inward or outward refers to the direction of the phase velocity in the frame of the unperturbed flow; the inward mode propagates inward or outward relative to the star, as $v-a^{2} / v$ is $<$ or $>U$, respectively. The inward mode phase velocity vanishes at the CAK critical point. The ratio of velocity amplitude to density amplitude for both modes is given by $v^{\prime} /\left(\rho^{\prime} / \rho\right)=v_{p}-v$, and therefore the velocity and density are in phase for the outward mode and out of phase for the inward mode. In most of the supersonic part of the wind $U$ is the same order as the flow velocity $v$, and is much larger than $a$. Then the phase velocity of the inward mode is approximately $v-U$, with $v^{\prime} /\left(\rho^{\prime} / \rho\right) \approx-U$, while the phase velocity of the outward mode is approximately $v+a^{2} / U$, with $v^{\prime} /\left(\rho^{\prime} / \rho\right)=a^{2} / U$. That is, the inward mode propagates inward supersonically relative to the mean flow, with a large velocity fluctuation, while the outward mode propagates outward quite subsonically relative to the mean flow, with a negligible velocity fluctuation. In the subsonic region, however, $U$ becomes negligible, and the inward and outward modes are ordinary sound waves with $v^{\prime} /\left(\rho^{\prime} / \rho\right)= \pm a$.

When the departure from the Sobolev approximation is taken into account with the bridging law (4), as was done by Owocki and Rybicki (1984), the phase velocities of the modes are modified somewhat, even for small $k$, and the modes acquire a growth or decay 


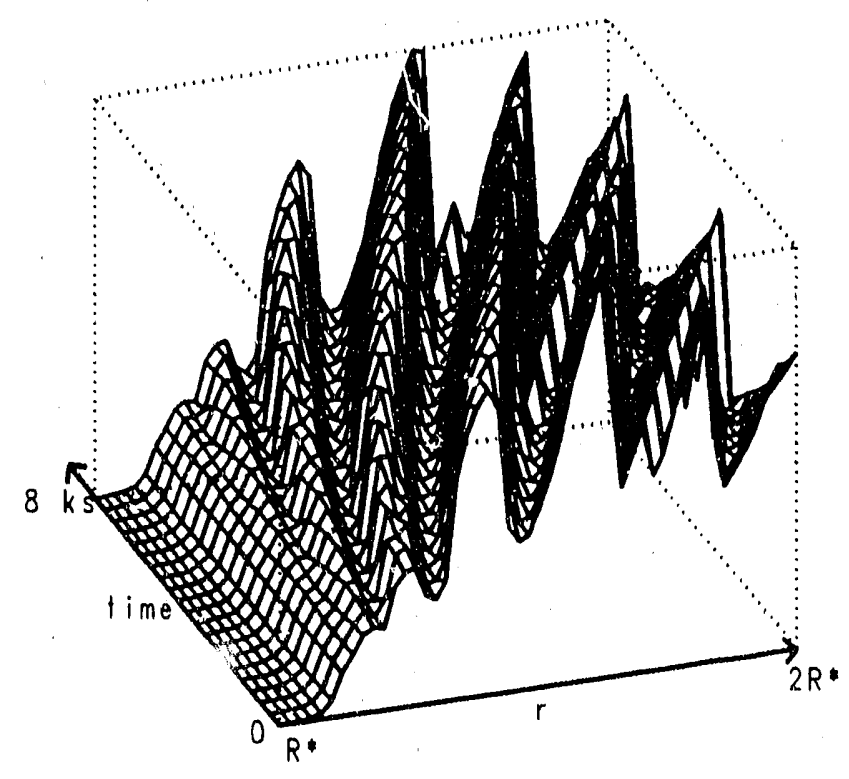

Figure 3: Like Fig. 1, witk $v_{\text {th }} / a=3 / 8$, but with the inner boundary driven by a sound wewe with $P=4000 \mathrm{~s}$ and $\rho^{\prime} / \rho=0.01$, after enough time has elapsed to make the motion periodic.

rate. It is then found that the irward mode has a large growth rate for outward propagation, and that its phase velocity exactly vanishes at the mean sonic point. It is now easy to describe the morphology of the advectively- and perhaps giobally-unstable wind: it is dominated by large amplitude inward-mode Abbott waves. The inward-racing nature of the strong rarefactions is natural for this mode. The compression side of the wave soon steepens into a. shock, which is the reverse shock of which many are seen in the simulations. The interaction between adjacent waves produces the forward shocks.

For the wind models that have $v_{\mathrm{th}} / a Z, 0.35$ the flow tends to a steady state in the absence of applied perturbations. Since in nature a complete absence of perturbations might be the exception rather than the rule, it is of interest to consider the response of such a model to an applied flux of sound waves. Figure 3 illustrates the behavior of the $v_{\text {th }} / a=3 / 8$ model of Fig. 1 when a sound wave with a period of $4000 \mathrm{~s}$ and an amplitude $\rho^{\prime} / \rho=0.01$ is incident at the lower boundary. It is apparent that exactly the same kind of waves are seen as in the unstable self-excited models, which were interpreted above as nonlinear inward-mode Abbott waves, with the only difference being that the oscillations are exactly periodic at the driving period.

A Fourier analysis of the temporial variations of density and velocity in this model leads to amplitudes and phases that can be compared with the linear theory. Exact agreement between nonlinear and linear results can not be expected, but the agreement with the inward mode properties is close enough to lend confidence to that identification. The phase velocity of the nonlinear waves in the simulation can be computed from the period and the radial derivative of the phase of the velocity variations; this is shown in Figure 4, where it is compared with the results of the linear dispersion analysis and with the unperturbed flow velocity. The numerical phase velocity agrees very closely with the outward mode in the 


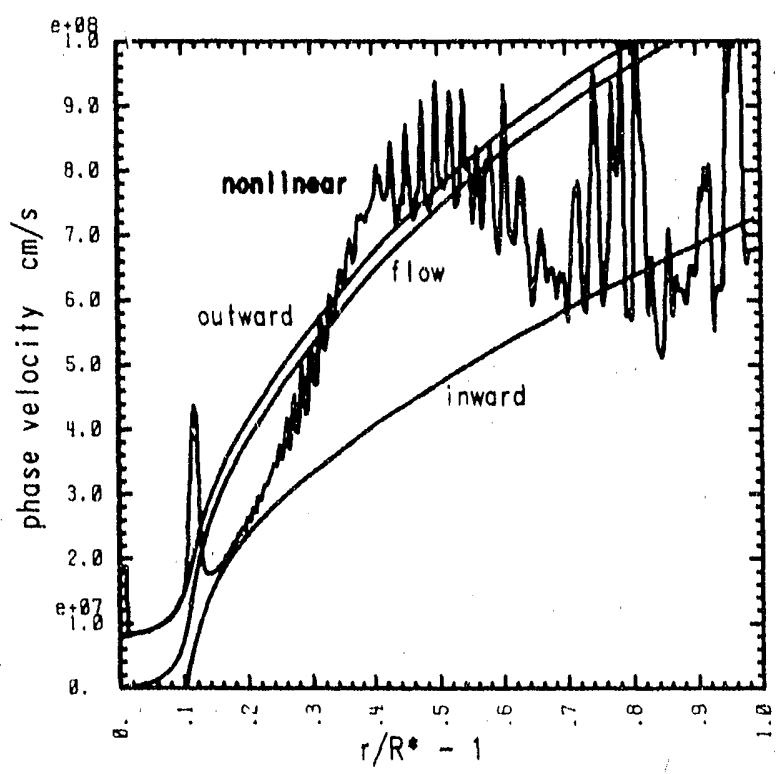

Figure 4: Phase velocity vs. radius for the $v_{\mathrm{th}} / a=3 / 8$ model driven as in Fig. 3. The curve labelled nonlinear is the numerical phase velocity obtained from the simulation. The inward and outward mode phase velocities based on a dispersion analysis of the steady model, and the steady flow velocity, are also shown.

subsonic region, where the wave modes correspond to sound waves. There is a "glitch" in the phase velocity as the sonic point is passed, and in the acceleration zone just outside the sonic point the phase velocity tends to follow that of the inward mode, until nonlinearity and numerical noise cause it to depart. The conclusion is that the driving at the lower boundary couples to the outward mode in the subsonic region, but that when the phase velocity of the inward mode changes from negative to positive at the sonic point, the driven oscillation also switches from outward mode to inward mode.

\section{Conclusions}

- The radiatively-driven stellar wind has a very strong linear instability. This is certainly true in the convective sense of Bers (1983), but also in the absolute sense in some cases.

- Hydrodynamic simulations of the unstable wind are very difficult, but possible; the radiation force must be severely approximated.

- The nonlinear behavior of wind simulations in the absorption approximation for the radiation force depends critically on the ratio of the width of the photospheric absorption profile to the sound speed. Narrow line $\rightarrow$ globally unstable wind; wide line $\rightarrow$ negligible wind.

- The unsteady wind is dominated by large-scale strong rarefactions and associated reverse shocks. 
- These waves correspond most closely with inward-mode radiation-acoustic (Abbott) waves.

- The high-frequency tail of the wave spectrum is not prominent in the nonlinear results, contrary to expectations based on the dependence of linear growth rate on $k$.

- The wave morphology has encouraging points of correspondence with observations of O-star winds, but difficulties remain, especially concerning the time dependence of the phenomena.

ACKNOWLEDGEMENTS. Portions of this work were supported by an Institutional Research and Development grant from Lawrence Livermore National Laboratory. Some of the calculations were carried out using an allocation of supercomputer time from the San Diego Supercomputer Center to S. P. Owocki. This work was a collaborative effort with S. P. Owocki and G. B. Rybicki, for whose assistance and advice I am very grateful.

\section{REFERENCES}

Abbott, D. C.: 1980, Astrophys. J. 242, 1183.

Bers, A.: 1983, in Handbook of Plasma Physics, Vol. 1: Basic Plasma Physics I, ed. A. A. Galeev and R. N. Sudan, North-Holland, Amsterdam, p. 451.

Cassinelli, J. P. and Olson, G. L.: 1979, Astrophys. J. 228, 304.

Cassinelli, J. P. and Swank, J. H. 1983, Astrophys. J. 271, 681.

Castor, J. I.: 1987, in Instabilities in Luminous Early Type Stars, ed. H. J. G. L. M. Lamers and C. W. H. de Loore, D. Reidel Publishing Co., Dordrecht, p. 159.

Castor, J. I., Abbott, D. C. and Klein, R. I.: 1975, Astrophys. J. 185, 157 (CAK).

Courant, R. and Friedrichs, K. O.: 1948, Supersonic Flow and Shock Waves, Interscience, New York, chapt. II.

Friend, D. B. and Abbott, D. C.: 1986, Astrophys. J. \$11, 701.

Hedstrom, G. W.: 1979, J. Comp. Phys. 30, 222.

Henrichs, H. F.: 1984, in Fourth Eumpean IUE Conference, ed. E. Rolfe and B. Battrick, ESA SP-218, p. 43.

Kaper, L., Henrichs, H. F., Zwarthoed, G. A. A. and Nichols-Bohlin, J.: 1990, in Angular Momentum and Mass Loss for Hot Stars, ed. L. A. Willson and R. Stalio, Kluwer, Dordrecht, p. 213.

Lamers, H. J. G. L. M., Gathier, R. and Snow, T. P.: 1982, Astron. Astrophys. 258, 186.

Lamers, H. J. G. L. M., Snow, T. P., de Jager, C. and Langerwerf, A.: 1988, Astrophys. J. 325, 342.

Lucy, L. B.: 1982, Astrophys. J. 255, 286.

Lucy, L. B.: 1984, Astrophys. J. 284, 351.

Lucy, L. B. and Solomon, P.: 1970, Astrophys. J. , 159, 879.

Lucy, L. B. and White, R. L.: 1980, Astrophys. J. 241, 300.

MacGregor, K. B., Hartmann, L. and Raymond, J. C.: 1979, Astrophys. J. 231, 514.

Milne, E. A.: 1926, Mon. Not. R. astr. Soc. 86, 459.

Morton, D. C.: 1967, Astrophys. J. 150, 535.

Owocki, S. P., Castor, J. I. and Rybicki, G. B.: 1988, Astrophys. J. 335, 914.

Owocki, S. P. and Rybicki, G. B.: 1984, Astrophys. J. 284, 337.

Owocki, S. P. and Rybicki, G. B.: 1985, Astrophys. J. 298, 265.

Owocki, S. P. and Rybicki, G. B.: 1986, Astrophys. J. 300, 127.

Pauldrach, A., Puls, J. and Kudritzki, R. P.: 1986, Astron. Astrophys. 164, 86.

Poe, C. H., Owocki, S. P. and Castor, J. I.: 1990, Astrophys. J. 358, 199.

Prinja, R. K.: 1990, in Angular Momentum and Mass Loss for Hot Stars, ed. L. A. Willson and R. Stalio, Kluwer, Dordrecht, p. 223

Prinja, R. K. and Howarth, I. D.: 1986, Astrophys. J. Supp. Ser. 61, 357.

Prinja, R. K. and Howarth, I. D.: 1988, Mon. Not. R. astr. Soc. 233, 123.

van Leer, B.: 1977, J. Comp. Phys. 23, 276. 

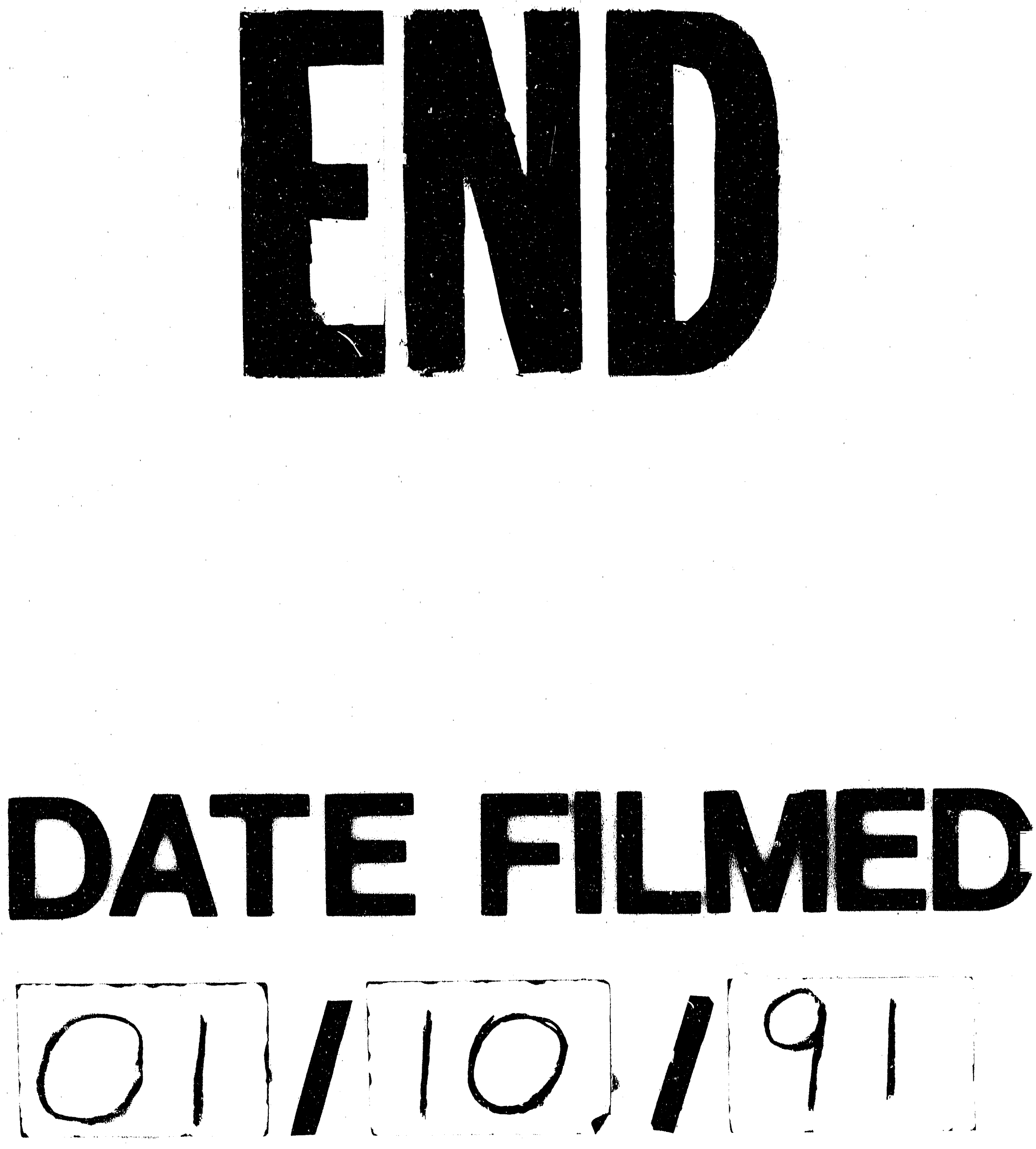
